\title{
Estrutura etária e mortalidade do linguado branco, Paralichthys patagonicus, (Jordan, 1889) no sul do Brasil
}

\author{
(Age structure and mortality of the white flounder Paralichthys patagonicus \\ (Jordan, 1889) in southern Brazil)
}

\author{
Júlio Neves de Araújo ${ }^{1} \&$ Manuel Haimovici ${ }^{2}$ \\ ${ }^{1}$ Fundação Universidade do Rio Grande - FURG \\ Curso de Pós-graduação em Oceanografia Biológica \\ ${ }^{2}$ Departamento de Oceanografia - FURG \\ e-mail: docmhm@super.furg,br \\ (Caixa Postal 474, 96201-900 Río Grande, RS, Brasil)
}

- Abstract: The age composition and mortality coefficients of the white flounder, Paralichthys patagonicus (Jordan, 1889), from southern Brazil were studied based on total length and age at length data from the trawl fishery landings in Rio Grande from 1989 to 1993 . Males were more frequent in the 30 to $40 \mathrm{~cm}$ length classes and females in the classes over $40 \mathrm{~cm}$. Recruitment occurs mainly at ages 2 and 3, and age groups 2 to 7 accounted for more than $90 \%$ of the weight of the catches. The instantaneous natural mortality coefficients $(M)$ were estimated from maximum observed ages as 0.35 and 0.42 for females and males, respectively. Average instantaneous total mortality coefficients $(Z)$ (1990-1993), estimated using catch curves, were 1.14 for males and 0.99 for females. Estimated instantaneous fishing mortality coefficients $(F=Z-M)$ were 0.72 for males and 0.64 for females and exploitation coefficients $(E=F / Z)$ were 0.63 and 0.65 , respectively. Exploitation rates over 0.5 indicate that the catches in the second half of the 1980 's were higher than those sustainable.

- Resumo: As composições de idades e os coeficientes instantâneos de mortalidade do estoque de linguado-branco, Paralichthys patagonicus (Jordan, 1889), explorado no sul do Brasil foram estimadas com base em composiçôes de comprimentos totais e de idades de amostragens de desembarques da pesca de arrasto de fundo no porto de Rio Grande entre 1990 e 1993, Os machos foram mais freqüentes nas classes de comprimentos entre 30 e $40 \mathrm{~cm}$ e as fêtmeas nas classes acima de $40 \mathrm{~cm}$. O recrutamento à pesca de arrasto ocorreu principalmente nas classes de idades 2 e 3 e as classes etárias 2 a 7 representaram mais de $90 \%$ das capturas em peso. Os eoeficientes instantâneos de mortalidade natural $(M)$ estimadas a partir das idades máximas observadas, foram 0,35 e 0,42 para fêmeas e machos respectivamente. Os coeficientes instantâneos de mortalidade total $(Z)$, estimadas a partir de curvas de captura foram, em média 1,14 para machos e 0,99 para fềmeas. Os coeficientes instantâneos de mortalidade por pesca $(F=Z-M)$, foram 0,72 para machos e 0,64 para fêmeas e as taxas de exploração $(E=F / Z) \quad 0,63$ e 0,65 respectivamente. As taxas de exploração superiores a 0,5 indicam que as capturas na segunda metade da década de 80 eram superiores às sustentáveis.

- Descriptors: Mortality, Age structure, Paralichthys patagonicus, Southern Brazil.

- Descritores: Mortalidade, Estrutura etăria, Paralichthys patagonicus, Sul do Brasil.

\section{Introdução}

O linguado branco, Paralichthys patagonicus (Jordan, 1889), é o pleuronectiforme mais intensamente explorado na plataforma continental do sul do Brasil (Haimovici \& Mendonça, 1996). Devido a sua importância eçonômica, vários estudos sobre sua distribuição e biologia foram 
realizados nesta região nos últimos anos. Este linguado se alimenta de crustáceos e peixes quando juvenil e subadulto e essencialmente de peixes na fase adulta. (Kawakami, 1975; Carneiro, 1995). Apresenta um período reprodutivo prolongado, com desova parcelada entre a primavera e fins do verão, com um máximo no mês de novembro. A primeira maturação sexual das fêmeas ocorre aos $33 \mathrm{~cm}$ de comprimento total e dos machos aos $31 \mathrm{~cm}$ (Carneiro, op. cit.). As idades podem ser determinadas sobre os otólitos. As fêmeas são mais longevas e atingem maiores tamanhos que os machos (Araújo \& Haimovici, 2000).

A pesca de linguados marinhos no sul do Brasil iniciou-se no final da década de 1940 junto com a pesca de arrasto de fundo (Yesaki \& Bager, 1975). Entre 1975 a 1985 foram desembarcados em Rio Grande, em média, 409 toneladas anuais (Haimovici et al., 1989). Nos anos seguintes ocorreu um aumento significativo dos desembarques com o início da pesca de arrasto duplo de tangones até atingir um máximo de $2.157 \mathrm{t}$ em 1989, mas em anos recentes os desembarques têm diminuído (Barcellos et al. 1991; Haimovici, 1998). Embora menos abundante que outros teleósteos demersais da região, por seu valor econômico, está entre os principais recursos pesqueiros do sul do Brasil.

Junto com os parâmetros reprodutivos e de crescimento, as taxas de mortalidade são utilizadas como dados básicos em diferentes modelos de dinâmica populacional empregados na avaliação do estado de exploração de recursos pesqueiros. Portanto, este trabalho teve como objetivos estimar as composições de idades e as taxas de mortalidade do linguado branco no litoral sul do Brasil, com base nos dados obtidos no início da década de 1990.

\section{Material e métodos}

Os dados utilizados neste estudo provêm da plataforma continental da região sul do Brasil entre as latitudes $30^{\circ} \mathrm{S}$ e $34^{\circ} \mathrm{S}$, em profundidades inferiores a 100 metros. As composições de comprimentos foram obtidas em amostragens de desembarques comerciais no porto de Rio Grande entre os anos de 1990 e 1993. Neste período, os locais de desembarque foram percorridos várias vezes por semana $\mathrm{e}$, nos casos em que foram encontrados arrasteiros de portas, parelha ou tangones descarregando linguado, foram coletados dados referentes à pescaria e realizadas medições de comprimento.

$\mathrm{Na}$ maior parte das vezes, foi retirada uma amostra ao acaso de 100 a 200 exemplares na saída dos cilindros lavadores antes da classificạ̧ão por tamanhos. Para evitar vícios de amostragem foram recolhidos todos os exemplares que passaram na esteira em vários periodos de alguns minutos, até completar a amostra (Haimovici, 1987). Em algumas situações, quando a descarga já havia terminado e o número de caixas de cada categoria de tamanhos de linguados conhecido, foram feitas amostragens estratificadas, de duas caixas de cada categoria de tamanhos (Gulland, 1966). De cada exemplar foi registrado o comprimento total $(C T)$ medido ao centímetro inferior e o sexo. Os linguados são desembarcados parcialmente eviscerados e o sexo pode ser determinado pela presença de parte das gônadas ou pelo formato da cavidade abdominal, que nas fêmeas se prolonga mais em direção à cauda.

As composições de idades das capturas desembarcadas foram estimadas a partir de chaves comprimento-idade, de composições de comprimentos nas amostragens dos desembarques e das relações comprimento total $\mathrm{x}$ peso eviscerado $(P E)$, e equações de crescimento apresentadas em Araújo e Haimovici (2000):

$$
\begin{aligned}
& P E_{\text {fèmeas }}=0,261 \times 10^{-5} \times C T^{3,213} \\
& P E_{\text {machos }}=0,599 \times 10^{-5} \times C T^{3,081} \\
& P E_{\text {sexos agrupados }}=0,337 \times 10^{-5} \times C T^{3,172} \\
& C T_{t \text { fêmeas }}=623,0(1-\exp (-0,279(t+0,243))) \\
& C T_{t \text { machos }}=534,1(1-\exp (-0,214(t+1,589))) \\
& C T_{t \text { sexos agrupados }}=609,1(1-\exp (-0,255(t+0,462))) \\
& \left.P E_{t \text { fêmeas }}=2488 \times(1-\exp (-0,279 \times(t+0,243)))\right)^{3,213} \\
& P E_{t \text { machos }}=1493 \times(1-\exp (-0,214 \times(t+1,589)))^{3,081} \\
& P E_{t \text { sexos agrupados }}=2294 \times(1-\exp (-0,255 \times(t+0,462)))^{3,172}
\end{aligned}
$$

$O$ processo de mortalidade consiste na diminuição da abundância de uma coorte ao longo do tempo (Ricker, 1975; Gulland, 1983). Os coeficientes instantâneos de mortalidade total $Z$, foram estimadas a partir de curvas de captura para intervalos de idades iguais e desiguais (Jones, 1984; Sparre et al., 1989).

As proporções das diferentes idades nas capturas foram estimadas a partir das composições de comprimentos nos desembarques de arrasteiros de parelha e tangones (Fig. 1) e de chaves comprimentoidade e são apresentadas na Figura 2.

Para intervalos de tempo iguais, os valores de $Z$ foram calculados através da equação $\ln N_{t}=a-$ $Z t$, onde $N_{t}$ eram valores proporcionais aos números de indivíduos por idades $t$ em anos. Para intervalos de tempo desiguais foi utilizado o método proposto por Pauly (1983). As idades relativas foram derivadas da equação inversa de von Bertalanffy, $t^{\prime}=t_{0}-(1 / K) \ln$ (1 - $\left.C T_{t} / C T_{\infty}\right)$, onde $C T_{t^{\prime}}$ é o ponto médio de cada intervalo de comprimento e $t^{\prime}$ a idade relativa em anos correspondente, e os valores de $K$ e $C T_{\infty}$ foram os das equações de crescimento do linguado branco no período considerado. Os valores de $Z$ foram calculados através da equação $\ln \left(N_{t} / \Delta t^{\prime}\right)=a-Z t^{\prime}$, onde $\Delta t^{\prime}$ é o tempo médio de crescimento correspondente a uma determinada classe de comprimento e que aumenta com o tamanho dos peixes. 


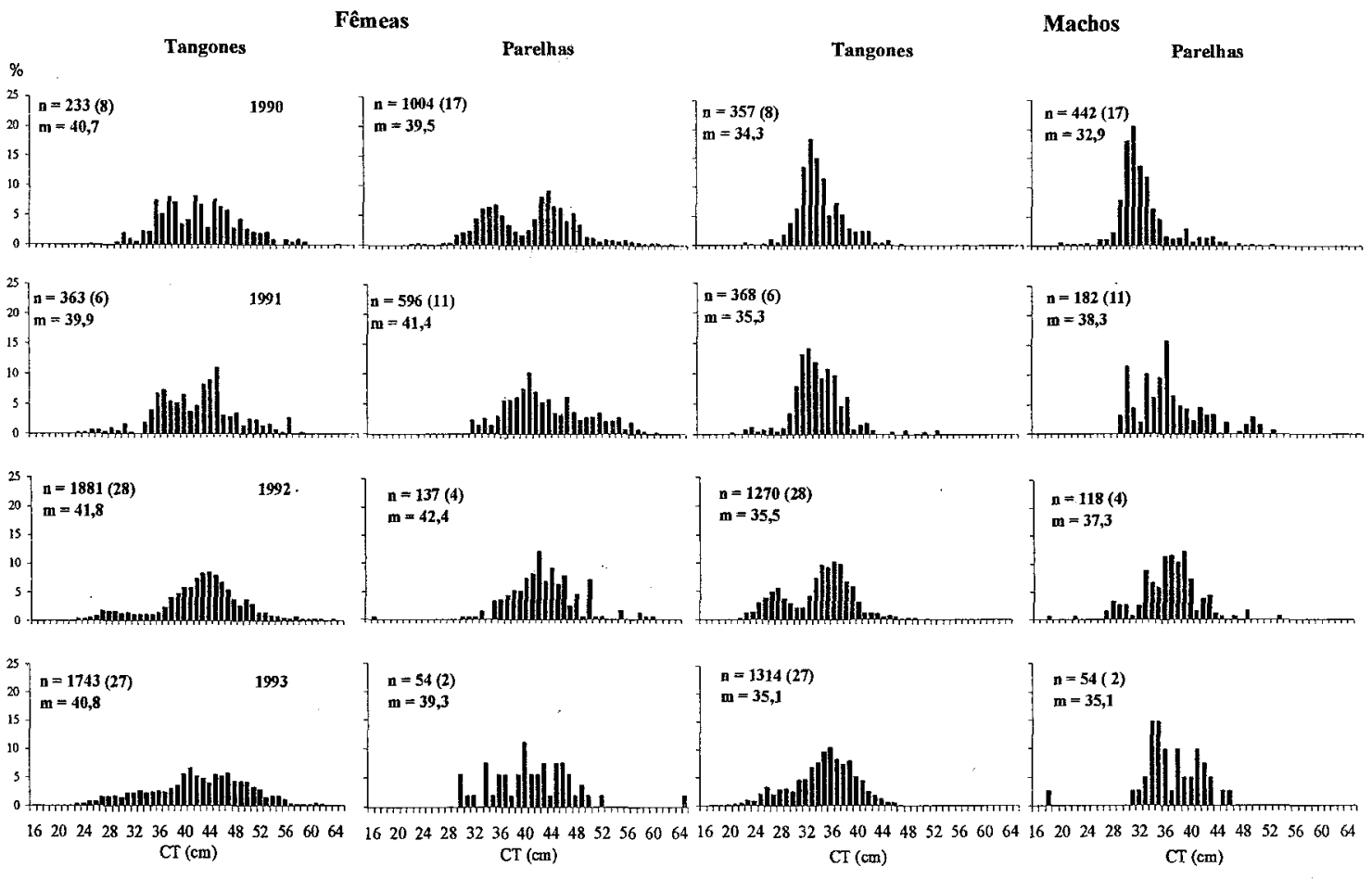

Fig. 1. Composições de comprimentos de machos e fềmeas de Paralichthys patagonicus nas amostras de desembarques de tangones e parelhas realizados em Rio Grande entre 1990 e 1993 ( $n=$ total de exemplares medidos; entre parênteses número de amostras; $\mathrm{m}=C T$ médio).

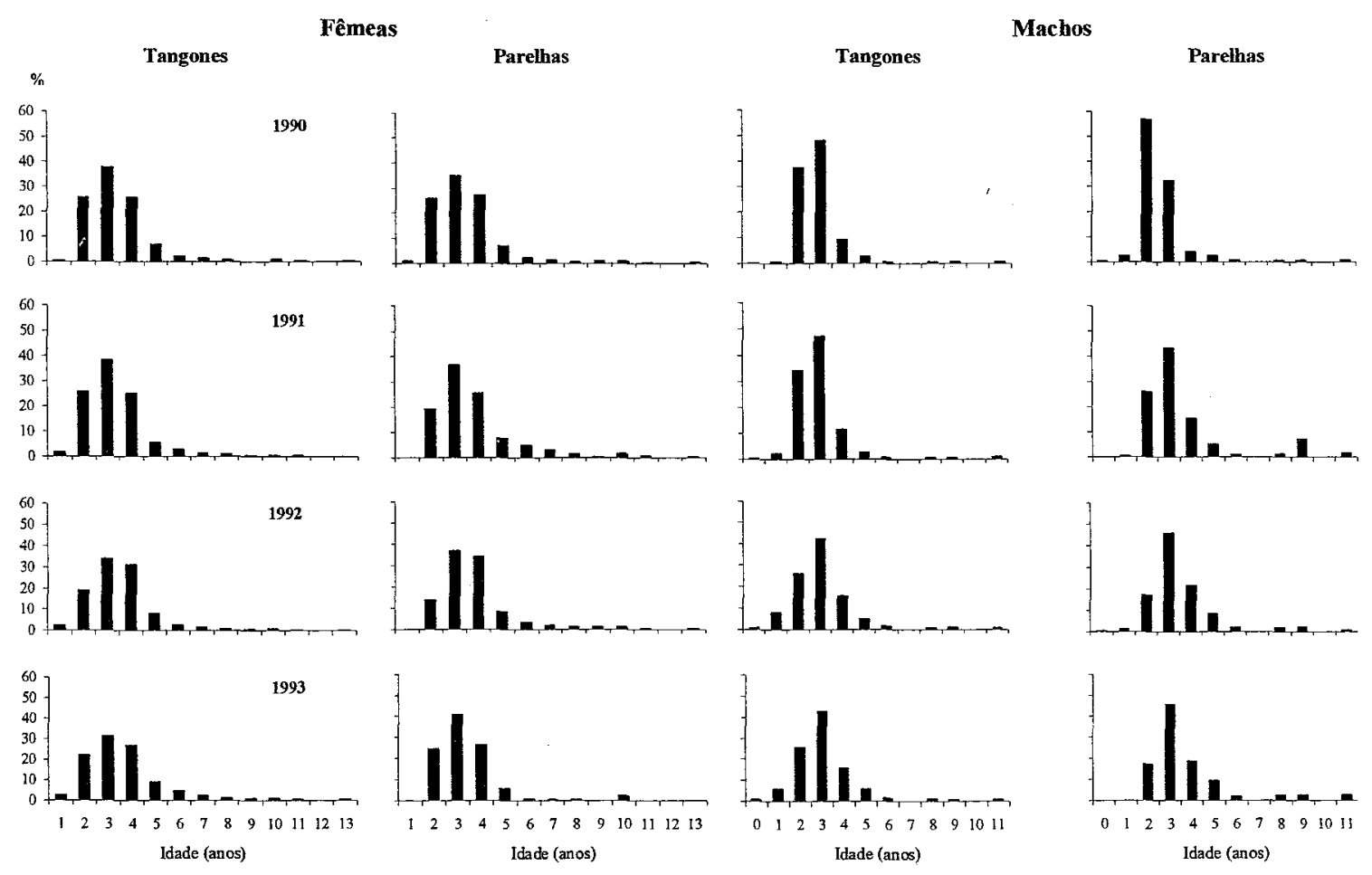

Fig. 2. Composição de idades de Paralichthys patagonicus nas amostras das capturas de tangones e parelhas desembarcadas em Rio Grande no período de 1990 a 1993. 
A mortalidade natural engloba diversas causas como condições abióticas adversas, falta de alimento, doença, senilidade e predação (Ricker, 1975). A taxa instantânea de mortalidade natural, $M$ foi estimada pelo método de Alagaraja (1984) que define como longevidade $t_{\text {máx }}$ a idade em anos com a qual entre $99 \%$ e $99,9 \%$ de uma coorte recrutada a pesca teria morrido se fora exposta somente à mortalidade natural. Portanto, se os valores de $t_{\text {máx }}$ representam sobrevivências de $1 \%$ a $0,1 \%$ dos peixes que se recrutam à arte de pesca, Os coeficientes instantâneos de mortalidade correspondentes podem ser calculadas como:

$M_{1 \%}=\ln (0,01) / t_{m \dot{\alpha} x}$ e $M_{0,1 \%}=\ln (0,001) / t_{m \dot{x} x}$

\section{Resultados}

\section{Composições de comprimento e idade}

No período 1990-1993 foram realizadas 103 amostragens de comprimentos nos desembarques de arrasteiros de parelhas e tangones, nas quais foram medidos 10.103 linguados (Tab. 1). As chaves comprimento-idade foram construídas a partir das leituras dos otólitos de 440 linguados amostrados em 1990 e 1991 e que também foram utilizadas no estudo do crescimento (Araújo \& Haimovici, 2000).

As composições de comprimentos de machos e fềmeas de Paralichthys patagonicus nọ desembarques de parelhas e tangones realizados em Rio Grande no período 1990 a 1993 estão apresentadas na Figura 1. Os machos foram mais freqüentes nas classes entre $30 \mathrm{e} 40 \mathrm{~cm}$ e as fềmeas na classes acima de $40 \mathrm{~cm}$. O maior macho mediu 54 $\mathrm{cm}$ e a maior fềmea $65 \mathrm{~cm}$. Os comprimentos médiọs anuais de machos variaram de 32,9 a $38,3 \mathrm{~cm}$ e os das fềmeas de 39,5 a 42,4 cm, mas não ficou configurada nenhuma tendência de mudança consistente neste parâmetro (Tab. 1).

As composições de idade por sexos dos linguados nas amostras anuais dos desembarques de parelhas e de tangones são apresentadas na Figura 2. Em ambos os sexos as idades dois a seis representaram em média mais de $90 \%$ da captura em número $\mathrm{e}$ as idades dois a sete representaram em média aproximadamente $90 \%$ da captura em peso.

\section{Mortalidade total}

As curvas de captura dos desembarques de arrasteiros de parelha e de tangones por anos são apresentadas na Figura 3. Os machos de idades até dois anos e as fêmeas até três não se recrutaram completamente nas capturas desembarcadas e os machos de idades superiores a cinco e fêmeas de idades superiores a sete foram pouco freqüentes. Portanto, as taxas de mortalidade total, calculadas como as inclinações das curvas de captura, incluíram apenas as idades três a cinco dos machos e quatro a sete das fêmeas. Os valores estimados de $Z$ anuais de machos variaram de 0,79 a 1,46, das fêmeas de 0,69 a 1,63 e dos sexos agrupados de 0,81 a 1,77 (Tab. 2). Houve uma considerável variabilidade nas estimativas da mortalidade total entre anos, reflexo das diferenças nas composições de comprimentos nos desembarques. Para os machos ocorreu uma tendência decrescente ao longo dos anos nos coeficientes de mortalidade estimados a partir das capturas com ambas as artes consideradas. Para as fềmeas, não se detectou uma tendência consistente. Alguns dos valores extremos obtidos neste estudo, como os derivados das amostragens de parelhas no ano de 1993 podem ser atribuidos ao baixo número de amostras disponivel. As médias dos valores de $Z$ anuais, ponderadas pela raiz quadrada do número de amostras de cada arte e ano, foi de 1,14 para os machos, 0,99, para as fềmeas e 1,09 para sexos agrupados (Tab. 2).

Os valores de $Z$ estimadas através do método de Pauly (Fig. 4) para os machos variaram de 0,53 a 1,30. Para as fêmeas, variaram de 0,53 a 1,66 e para sexos agrupados variaram de 0,64 a 1,75 . As observações anteriores a respeito dos erros amostrais relacionados ao baixo número de amostras são válidos também para este método e os $\mathrm{Z}$ médios ponderados foram de 0,99 para os machos, 0,90 para as fêmeas e 0,93 para sexos agrupados (Tab. 2).

\section{Mortalidade natural}

A maior idade de machos observada nas amostras foi de onze anos e menos de $1 \%$ destes atingiu a idade nove, correspondendo às estimativas de $M$ de 0,42 a 0,51 . A maior idade das fềmeas foi treze anos e menos de $1 \%$ atingiu a idade dez, correspondendo às estimativas de 0,35 a 0,46 . Como a pescaria de linguado-branco no sul do Brasil já era bastante intensa em 1985 (Barcellos et al., 1991) as $M$ derivadas das maiores idades observadas nas capturas foram considerados como as melhores estimativas dos coeficientes instantâneos de mortalidade natural, ou seja, 0,35 para as fêmeas e 0,42 para os machos.

\section{Mortalidade por pesca e taxa de exploração}

Os coeficientes instantâneos de mortalidade por pesea $(F)$ foram calculadas por diferença entre as taxas de mortalidade totais médias estimadas e as taxas de mortalidade natural. As taxas de exploração $(E)$ foram calculadas como os quocientes dos coeficientes médios de mortalidade por pesca e total. Como não se observaram tendências nítidas de mudança entre anos e artes de pesca nas taxas de mortalidade total estimadas por ambos métodos (Tab. 2), obtiveram-se estimativas únicas para o período 1990 a 1993. 
Tabela 1. Comprimentos e pesos médios do linguado branco Paralichthys patagonicus nas amostragens de desembarques de tangones e parelhas realizados em Rio Grande no período de 1990 a 1993 . CT $=$ comprimento total em milímetros; $\mathrm{PE}=$ peso eviscerado em gramas.

\begin{tabular}{lcccccccc}
\hline & \multicolumn{3}{c}{ TANGONES } & \multicolumn{4}{c}{ PARELHAS } \\
& 1990 & 1991 & 1992 & 1993 & 1990 & 1991 & 1992 & 1993 \\
\hline Número de amostras & 8 & 6 & 28 & 27 & 17 & 11 & 4 & 2 \\
Número de peixes medidos & 590 & 731 & 3151 & 3057 & 1446 & 778 & 255 & 95 \\
CT médio machos & 343 & 353 & 355 & 351 & 329 & 383 & 373 & 351 \\
CT médio fềmeas & 407 & 398 & 418 & 408 & 395 & 410 & 424 & 393 \\
PE médio machos & 426 & 470 & 484 & 469 & 393 & 621 & 560 & 479 \\
PE médio fềmeas & 723 & 676 & 776 & 746 & 682 & 739 & 804 & 655 \\
\% de machos & 60,5 & 50,3 & 40,3 & 43,0 & 30,6 & 23,4 & 46,2 & 43,2 \\
\% de fềmeas & 39,5 & 49,7 & 59,7 & 57,0 & 69,4 & 76,6 & 53,8 & 56,8 \\
\hline
\end{tabular}

Fêmeas
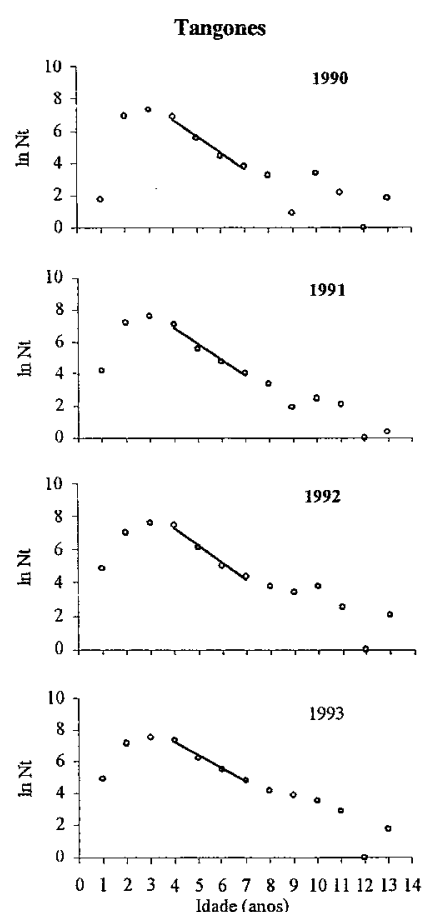

Idade (anos)
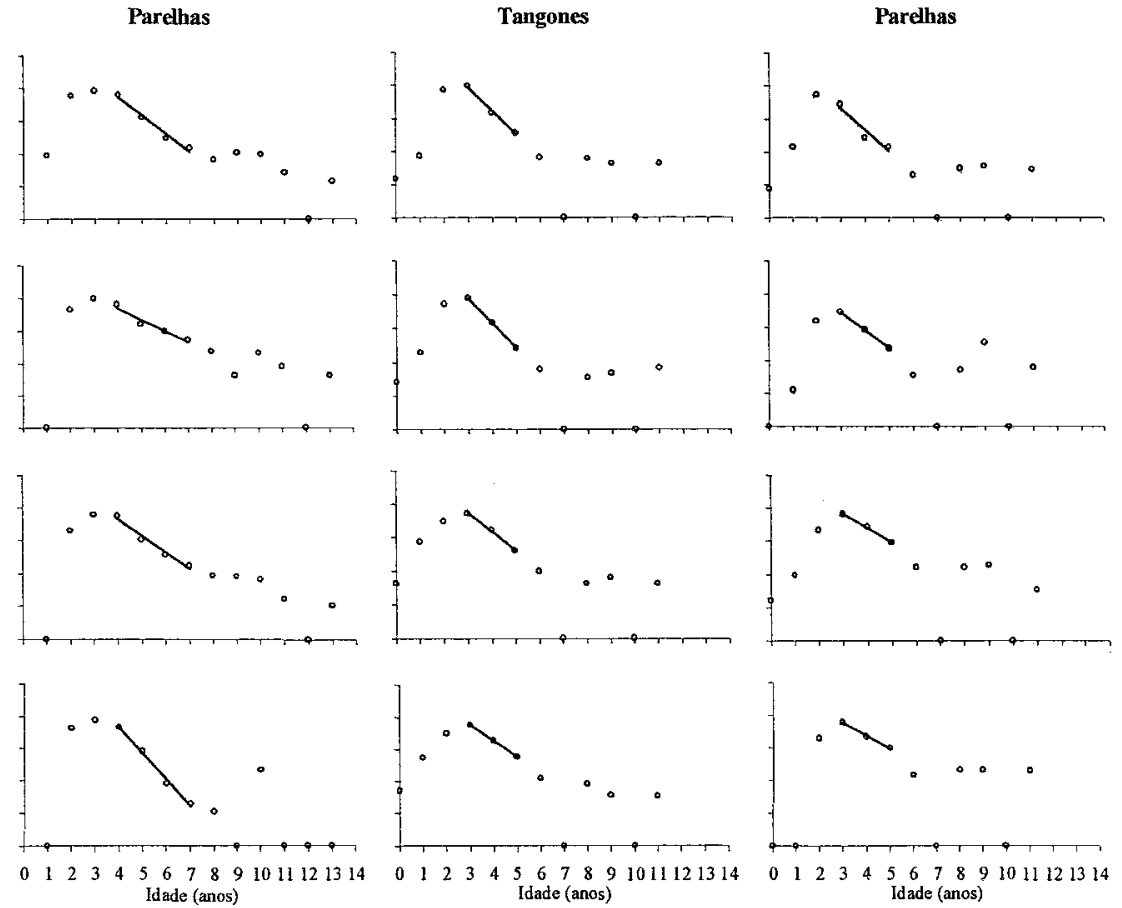

Fig. 3. Curvas de captura de Paralichthys patagonicus em base as amostras das capturas de tangones e parelhas desembarcadas em Rio Grande no período de 1990 a 1993. 
Tabela 2. Coeficientes instantâneos de mortalidade total $(Z)$ do linguado branco Paralichthys patagonicus estimadas em base as amostras de composição de comprimentos dos desembarques de tangones (T) e parelhas (P) em Rio Grande no período 1990-93, a partir dos métodos de curvas de captura para intervalos de tempo iguais e desiguais (ver no texto). $r=$ coeficiente de correlação linear; $t=$ idade observada em anos $t^{\prime}=$ idade






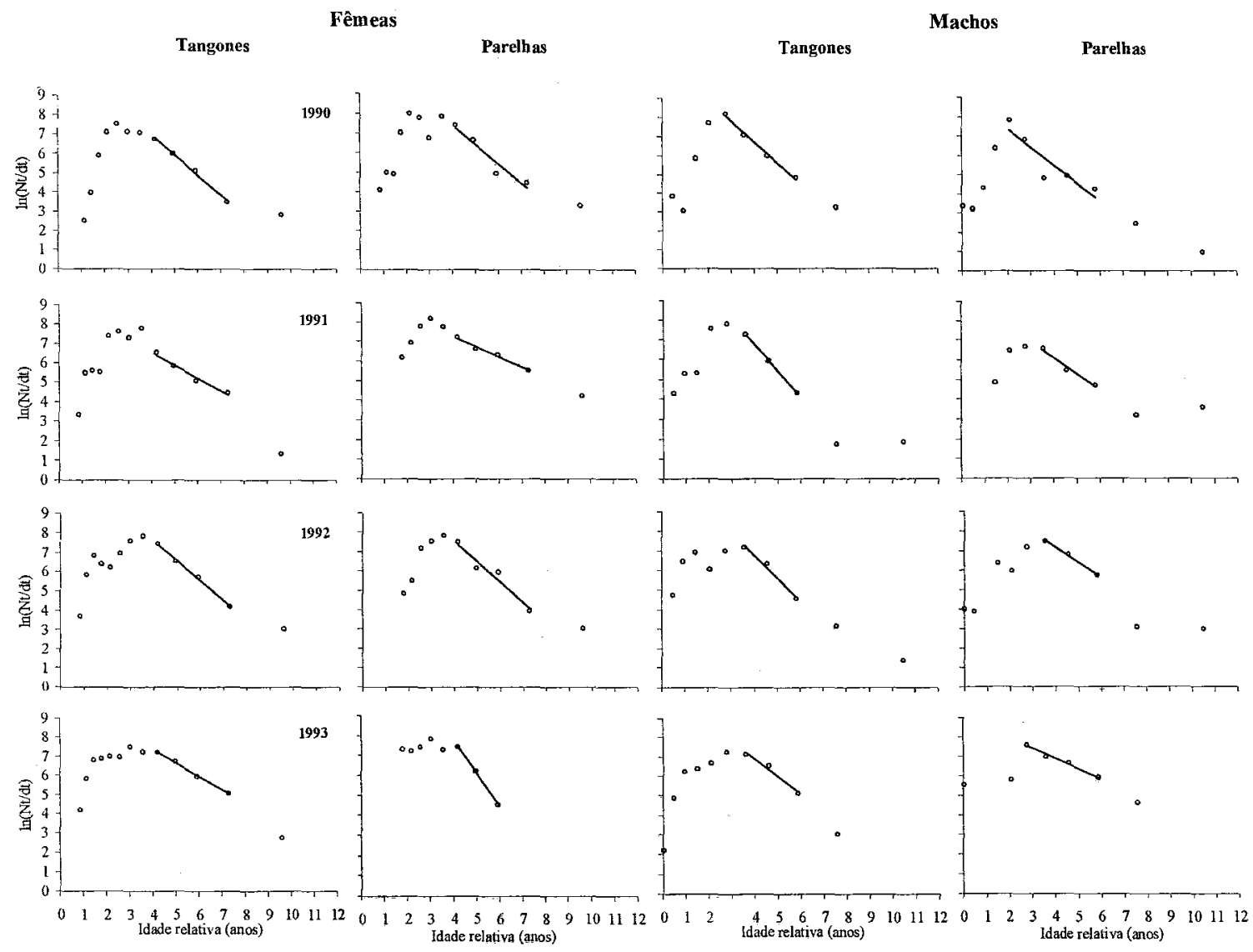

Fig. 4. Curvas de captura de comprimentos (método de Pauly) de Paralichthys patagonicus com base nas amostras das capturas de tangones e parelhas desembarcadas em Rio Grande no período de 1990 a 1993.

Tabela 3. Coeficientes instantâneos de mortalidade por pesca e taxas de exploração do linguado branco Paralichthys patagonicus no sul do Brasil, calculadas com base nas estimativas de mortalidade total obtidas de curvas de captura para intervalos de idades iguais (A) e desiguais (B) entre 1990 e 1993, e das estimativas de mortalidade natural obtidas pelo método de Alagaraja (ver texto).

\begin{tabular}{lcccc}
\hline & \multicolumn{2}{c}{ MACHOS } & \multicolumn{2}{c}{ FÊMEAS } \\
& A & B & A & B \\
\hline Mortalidade natural $\left(\mathrm{M}\right.$, ano $\left.^{-1}\right)$ & 0,42 & 0,42 & 0,35 & 0,42 \\
Mortalidade total $\left(\mathrm{Z}\right.$, ano $\left.^{-1}\right)$ & 1,14 & 0,99 & 0,99 & 0,90 \\
Mortalidade por pesca $\left(\mathrm{F}\right.$, ano $\left.^{-1}\right)$ & 0,72 & 0,57 & 0,64 & 0,55 \\
Taxa de exploração $\left(\mathrm{E}\right.$, ano $\left.^{-1}\right)$ & 0,63 & 0,58 & 0,65 & 0,61 \\
\hline
\end{tabular}

As taxas de mortalidade por pesca e de exploração médias anuais estimadas a partir das curvas de capturas por idades foram $F=0,72 \mathrm{e}$ $E=0,63$ para machos e $F=0,64$ e $E=0,65$ para fêmeas. As taxas de mortalidade por pesca e de exploração médias estimadas a partir das curvas de captura de comprimentos foram $F=$ 0,57 e $E$.

\section{Discussão}

Os coeficientes instantâneos de mortalidade natural estimadas para Paralichthys patagonicus, de 0,35 para as fêmeas e 0,42 para machos são comparáveis com a estimativa de 0,40 para sexos agrupados de $P$. lethostigma, que apresenta parâmetros de crescimente, tamanho e idade de 
maturação sexual similares aos de $P$. patagonicus (Matlock, 1991; Miller et al., 1991) e bem próximas às estimadas para o linguado Lepidorhombus boscii (Santos, 1994) que foram de 0,41 para machos e 0,34 para fềmeas. Estas taxas são relativamente altas quando comparadas com as estimativas de $M$ de outros teleósteos da região com idades máximas acima de 20 anos como Umbrina canosai $(0,15$ a 0,25 ) (Haimovici, 1988) e idades máximas maiores de 30 anos como para Netuma barba $(0,12$ a 0,16$)$ (Reis, 1986), mas se encontram dentro da mesma faixa de valores das estimavas para Merluccius hubbsi $(0,30$ a 0,58) (Bezzi et al., 1994), cuja idade máxima observada foi 13 anos.

As taxas de exploração $E$ estimadas entre 0,58 e 0,63 para machos e 0,61 e 0,65 para fêmeas foram elevadas e indicam que as capturas no patamar da segunda metade da década de 1980 já não eram sustentáveis, já que valores de $E$ acima de 0,5 são considerados como indicadores de sobreexploração (Gulland, 1983). Estes resultados corroboram as conclusões de Haimovici \& Mendonça (1996) e Haimovici (1998), segundo as quais as capturas de linguados na região Sul, no período citado, apresentaram uma tendência de queda, embora não se observasse um aumento de esforço indicando que o estoque estava sendo explorado além de sua capacidaḍe de reposição sustentável,

As estimativas de $Z$ de machos a partir de curvas de captura de idades deixam em aberto a possibilidade de uma tendência de redução da mortalidade. Uma diminuição nas inclinações de uma seqüência de curvas de captura pode refletìr diversos processos como redução gradativa da mortalidade natural, no recrutamento ou no esforço de pesca (Ricker, 1975). Sobre os processos de redução da mortalidade natural e recrutamento não se dispõe de dados. Em relação à segunda hipótese, de redução no esforço, esta é pouco provável. ja que os desembarques de linguados aumentaram até 1989 sugerindo que o esforço se manteve ou aumentou até esse ano (Haimovici \& Mendonça, 1996; Haimovici, 1998).

Embora não seja possível compará-las estatisticamente, as taxas de mortalidade total estimadas a partir de curvas de captura de idade e de comprimento foram aproximadas e as taxas de exploração calculadas com base nessas estimativas também o foram. A vantagem das curvas de captura por comprimento para se estimar $Z$ reside em diminuir o esforço na obtenção, preparação e leitura de um número elevado de otólitos necessários para a construção de chaves comprimento-idades. Este método pode representar uma boa ferramenta para a estimativa da mortalidade total de $P$. patagonicus, desde que as possíveis mudanças de crescimento sejam monitoradas através de amostras relativamente pequenas, de 100 a 150 exemplares por sexo.

\section{Referências bibliográficas}

Alagaraja, K. 1984. Simple methods for estimation of parameters for assessing exploided fish stocks. Indian J. Fish., 31:177-208.

Araújo, J. N. \& Haimovici, M. 2000. Determinação de idades e crescimento do linguado-branco Paralichthys patagonicus (Jordan, 1889) no Sul do Brasil. Rev. bras. oceanogr., 48(1):

Barcellos, L. J. P.; Peres, M. B., Wahrlich, R. E Barison, M. B. 1991. Relatório sobre otimização bioeconômica dos recursos pesqueiros marinhos do Rio Grande do Sul. Rio Grande, FURG/Museu Oceanográfico. 58p.

Carneiro, M. H. 1995. Reprodução e alimentação dos linguados Paralichthys patagonicus e $P$. orbignyanus (Pleuronectiformes:Bothidae), no Rio Grande do Sul, Brasil. Dissertação de mestrado. Rio Grande. FURG. 80p.

Gulland, J. A. 1966. Manual de metodos de muestreo y estatisticos para la biologia pesquera. Parte 1: Metodos de muestreo. FAO. Fish. Div. 1v.

Gulland, J. A. 1983 Fish stock assessment: A manual of basic methods. FAO/Wiley ser. Food. Agric., $1: 1-223 p$.

Haimovici, M. 1987. Estratégia de amostragem de comprimentos de teleósteos demersais nos desembarques da pesca de arrasto no litoral Sul do Brasil. Atlântica, Rio Grande, 9(1):65-82.

Haimovici, M. 1988. Análisis de cohortes del stock de pargo blanco (Umbrina canosai) explotado en el sur de Brasil, Uruguay y Argentina. Com. Téc. Mix. Fr. Mar., 4:33-40.

Haimovici, M. 1998. Present state and perspectives for the southern Brazil shelf demersal fisheries. Fișh. Mange. Ecol., 5(4):277-289.

Haimovici, M. \& Mendonça J. T. 1996. Análise da pesca de arrasto de tangones de peixes e camarões no Sul do Brasil, no período 1989-1994. Atlântica, Rio Grande, 18:143-160.

Haimovici, M.; Pereira, S. D. \& Vieira, P. C. 1989. La pesca demersal en el sur de Brasil en el periodo 1975-1985. Frente Maritimo, 5:151-163.

Jones, R. 1984. Assessing the effects of in explotation pattern using lenth composition data (with notes on VPA and cohort analysis). FAO Fish. Tech. Pap., 256:1-118 . 
Kawakami, E. 1975. Alimentação de pleuronectiformes (análise comparativa e bionomia). Dissertação de mestrado. Universidade de São Paulo, Instituto Oceanográfico. $150 \mathrm{p}$.

Matlock, G. C. 1991. Growth, mortality, and yield of southern flounder in Texas. Northeast Gulf Science, 12 (1):61-65.

Miller, J. M.; Burke J. S. \& Fitzhugh, G. R. 1991. Early life history patterns of Atlantic North american flatfish: likely (and unlikely) factors controlling recruitment. Neth. J. Sea Res., 27(3/4):261-275.

Pauly, D. 1983. Length-converted catch curves. A pawerful tool for fisheries research in the tropics. ICLARM Fishbyte, 1(2):9-13.

Reis, E. G. 1986. Age and growth of the marine catfish, Netuma barba (Siluriformes, Ariidae), in the estuary of the Patos Lagoon (Brazil). Fish. Bull., 84(3):679-686.
Ricker, W. E. 1975 Computation and interpretation of biological statistics of fish populations. Bull. Fish. Bd Can., 191:1-382.

Santos, P. T. 1994. Growth and reproduction of the population of the four-spot megrin (Lepidorhombus boscii Risso) off the Portuguese Coast. Neth. J. Sea Res., 32(3-4):379-383.

Sparre, P.; Ursin, E. \& Venema, S. C. 1989. Introduction to tropical fish stock assessment. Part 1: manual. FAO Fish. tech. Pap., 306 (1):1-337.

Yesaki, M. \& Bager, K. J. 1975. Histórico da evolução da pesca industrial no Rio Grande. Sér. Doc. Técnicos, 11:1-15.

(Manuscrito recebido 13 agosto 1999; revisado 21 fevereiro 2000; aceito 24 abril 2000) 